\title{
International Journal of Medical Sciences
}

\section{Predictors of hepatic steatosis in HBeAg-negative chronic hepatitis B patients and their diagnostic values in hepatic fibrosis}

\author{
Rui-dan Zheng ${ }^{1}$, Cheng-run Xu ${ }^{1}$, Li Jiang ${ }^{1}$, Ai-xia Dou ${ }^{2}$, Kun Zhou ${ }^{2}$, Lun-gen Lu ${ }^{凶}$ \\ 1. Research and Therapy Center for Liver Diseases, Southeast Hospital, Zhangzhou 363000, China \\ 2. Department of Gastroenterology, Shanghai First People's Hospital, Shanghai Jiaotong University School of Medicine, \\ Shanghai 200080, China
}

$\triangle$ Corresponding author: Lun-Gen Lu, M.D., Department of Gastroenterology, Shanghai First People's Hospital, Shanghai Jiaotong University School of Medicine, Shanghai 200080, China. Tel: +86-21-63240090; Fax: +86-21-63241377; E-mail:live.0000@live.cn

Received: 2010.05.05; Accepted: 2010.08.08; Published: 2010.08.11

\begin{abstract}
Objective: To investigate predictors of hepatic steatosis in $\mathrm{HBeAg}$-negative chronic hepatitis $\mathrm{B}(\mathrm{CHB})$ patients and their diagnostic values in hepatic inflammation and fibrosis. Methods: $\mathrm{A}$ total of $106 \mathrm{HBeAg}$-negative $\mathrm{CHB}$ patients with clinically and pathologically proven steatosis and 98 patients without steatosis were recruited into this study. The levels of fasting blood glucose (FBG), fasting insulin (FINS), triglyceride (TG), cholesterol ( $\mathrm{CHOL})$, alanine aminotransferase (ALT), aspartate aminotransferase (AST), albumin (Alb), globulin (Glb), HBV DNA, body mass index (BMI), homeostatic model assessment of insulin resistance (HOMA-IR) and pathological changes of the liver in inflammation, fibrosis and fatty deposition were examined in all patients. Results: The levels of BMI, HOMA-IR, FBG, insulin, TG, and $\mathrm{CHOL}$ were significantly higher in patients with steatosis than those without steatosis (all $P<0.05)$. But ALT, AST and HBV DNA levels were significantly lower in patients with steatosis (all $P<0.05$ ). Logistic regression analysis showed that only FINS was a significant predictor for hepatic steatosis $(P<0.05)$; FINS and Glb were significant predictors for hepatic inflammation (all $P<0.05$ ); BMI and TC were significant predictors for hepatic fibrosis (all $P<0.05$ ). Conclusions: Hepatic steatosis, a common disease in $\mathrm{HBeAg}$-negative $\mathrm{CHB}$ patients, was positively associated with BMI, FBG, FINS, TG, TC, GGT, ALP and HOMA-IR. In these patients, the prevalence of hepatic inflammation and fibrosis was also increased.
\end{abstract}

Key words: HBeAg negative, chronic hepatitis B, nonalcoholic fatty liver disease, liver biopsy

\section{Introduction}

The prevalence of HBeAg-negative chronic hepatitis $B(\mathrm{CHB})$ tends to increase in recent years (1). With the improvement of living standard and nutrition status, hepatic steatosis frequently occurs in CHB patients. It has been shown that the incidence of hepatic steatosis in CHB patients was about $32 \%$ (2). The distribution of hepatic triglyceride content (HTGC) in 2, 287 subjects from a multiethnic, population-based sample $(32.1 \%$ white, $48.3 \%$ black, and $17.5 \%$ Hispanic) was previously examined and compared using proton magnetic resonance spectroscopy. Almost one third of the population had hepatic steatosis, and most subjects with hepatic steatosis had normal levels of serum alanine aminotransferase (ALT). The higher prevalence of hepatic steatosis in Hispanics was mainly due to the higher prevalence of obesity and insulin resistance in this ethnic group (3). But, that how does hepatic steatosis influence CHB still remains unclear (4), particularly in HBeAg-negative $\mathrm{CHB}$ patients. In contrast, a large body of evidence showed the incidence of hepatic steatosis in chronic hepatitis $\mathrm{C}(\mathrm{CHC})$ patients ranged from $31 \%$ to $72 \%$. Moreover, it has been suggested that hepatic steatosis in $\mathrm{CHC}$ has correlations with obesity, disorder of fat 
metabolism, insulin resistance and HCV genotypes (5). This study analyzed and compared the clinical and histological features of $\mathrm{HBeAg-negative} \mathrm{CHB}$ patients with or without hepatic steatosis, so as to evaluate the predictors of clinical and pathological characteristics in these patients with steatosis and their diagnostic values in hepatic fibrosis.

\section{Patients and methods}

\section{Patients}

A total of $204 \mathrm{HBeAg}$-negative $\mathrm{CHB}$ patients were recruited from the Research and Therapy Center for Liver Diseases of China Southeast Hospital from May 2005 to March 2009. Most patients did not drink alcohol, and alcohol consumption in the remaining patients was less than $20 \mathrm{~g} /$ day. These patients were divided in two groups according to presence of hepatic steatosis. Among all HBeAg-negative CHB participants, 106 were diagnosed as hepatic steatosis (83 men and 23 women, mean age: $41.08 \pm 10.23$ years) and the remaining 98 patients were excluded from hepatic steatosis (79 men and 19 women, mean age 39.4 \pm 9.81 years). Their diagnoses were finally confirmed by clinical presentations and pathological features. The criteria for hepatic steatosis were based on the American Association for the Study of Liver Diseases Practice Guidelines (2007) (6). Hepatitis A virus (HAV), $\mathrm{HCV}$, Hepatitis D virus (HDV) and Hepatitis E virus (HEV) infection, drug-induced hepatitis, alcoholic hepatitis and autoimmune hepatitis were all excluded. Clinical data were obtained and recorded immediately after enrollment.

\section{Body mass index (BMI)}

BMI was calculated as the individual's body weight divided by the square of his or her height. According to the new BMI criteria for Asians by the regional office for the western pacific region of WHO (WHO Technical Report Series No. 894, WHO, Geneva, 2000), normal weight, overweight, Obese Class I and Obese Class II were defined by BMI= 18.5-22.9 $\mathrm{kg} / \mathrm{m}^{2}, \quad 23.0-24.9 \mathrm{~kg} / \mathrm{m}^{2}, \quad 25.0-29.9 \mathrm{~kg} / \mathrm{m}^{2}$ and $\geq 30$ $\mathrm{kg} / \mathrm{m}^{2}$, respectively.

\section{Serum markers of HBV}

HBV markers, including HBsAg, anti-HBs, anti-HBc, HBeAg and anti-HBe were measured by enzyme-linked immunosorbent assay (Livzon Group Reagent Factory, Guangdong, China).

\section{HBV DNA}

The HBV DNA level was determined by quantitative polymerase chain reaction (qPCR) (AcuGen HBV quantitative test; Biotronic Corp., Lowell, Mass.) with the fluorescent HBV DNA probes provided by the same company. Asymmetric primer 1 was 5'-TGTCTCGTGTTACAGGCGGGGT-3', asymmetric primer 2 was 5'-GAGGCATAGCAGCAGGA GAAGAG-3', and fluorescent primer was 5'-TCGCTGGAAGTGTCTGCGGCGT-3'.

\section{Serum assays}

Fasting blood was collected with a un-anticoagulated vacuum blood collection tube. Serum was separated by centrifugation at $4^{\circ} \mathrm{C}$ and stored in a sterile tube at $-40^{\circ} \mathrm{C}$ within $4 \mathrm{~h}$. The levels of fasting blood glucose (FBG), insulin, triglyceride (TG), cholesterol (CHOL), ALT, aspartate aminotransferase (AST), $\gamma$-glutamyltransferase (GGT), alkaline phosphatases (ALP), albumin (Alb) and globulin (Glb) were determined.

\section{Homeostatic model assessment of insulin resis- tance (HOMA-IR)}

HOMA-IR was calculated by means of the homeostasis model assessment (HOMA-R) previously described, where HOMA-R=insulin/(22.5 $\left.\mathrm{e}^{- \text {ln glucose }}\right)$ (7).

\section{Histological evaluation}

All 204 specimens from liver biopsy were 1.0 2.5 $\mathrm{cm}$ in length. Liver biopsy was performed to obtain the specimens under the guidance of ultrasound within 1 week after admission, using a needle with an internal diameter of $1.4 \mathrm{~mm}$ (Quick-Cut; Hakko. Company, Japan). Each specimen was longer than 1 $\mathrm{cm}$ and had more than 6 portal areas. Specimens were fixed in buffered formalin, embedded in paraffin, and stained with hematoxylin-eosin-safran and Masson's trichrome. Hepatic steatosis, stage of fibrosis and grade of disease activity were determined according to the Guidelines for the assessment and management of non-alcoholic fatty liver disease in the Asia-Pacific region (8). Microvesicular steatosis was also graded as: F0 ( $<5 \%$ hepatocytes with microvesicular steatosis), F1 (5 30\% hepatocytes involved), F2 (31 50\% hepatocytes involved), F3 (51 75\% involved) and F4 (>75\% hepatocytes involved). Fibrosis stage was defined as S0 (no fibrosis), S1 (mild fibrosis), S2 (moderate fibrosis), S3 (severe fibrosis), and S4 (cirrhosis), and grade of disease activity was defined as G0 (no activity), G1 (mild activity), G2 (moderate activity), and G3 (severe activity). All the sections were blindly and independently assessed by 3 pathologists and the results were processed by the Kappa concordance test. The inter- and intra-observer agreements were excellent. 


\section{Statistical analysis}

Data were analyzed with the SPSS 12.0 statistical package (SPSS Inc., Chicago, IL, USA). Baseline characteristics and anthropometric indices were expressed as means \pm standard deviation (SD) or percentage frequency, if necessary. The baseline characteristic and anthropometric indices were compared between $\mathrm{HBeAg}$-negative $\mathrm{CHB}$ patients with steatosis and those without steatosis by independent $t$ test for continuous variables and Chi-square test for categorical variables. A binary logistic regression model was used to determine predictors and their odds ratios for hepatic steastosis among $\mathrm{HBeAg}$-negative $\mathrm{CHB}$ patients. To screen the predictors for both hepatic inflammation and fibrosis stages, multivariate logistic regression models with adjustment for age and gender were employed. For all comparisons, two-tailed $\mathrm{P}$ values of less than 0.05 were considered statistically significant.

\section{Results}

A total of 106 patients were diagnosed as hepatic steatosis (83 men and 23 women, mean age: $41.08 \pm 10.23$ years), and 98 patients excluded from hepatic steatosis (79 men and 19 women, mean age $39.4 \pm 9.81$ years) served as controls. There was no significant difference in age or gender between both groups $(P>0.05)$. Clinical characteristics and some anthropometric indices of $\mathrm{HBeAg}$-negative $\mathrm{CHB}$ patients with or without steatosis are listed in Table 1. $\mathrm{HBeAg-negative} \mathrm{CHB}$ patients with steatosis had significantly higher levels of BMI, FBG, FINS, TG, TC, GGT, ALP, Glb and HOMA-IR (all $P<0.05$ ) than did those without steatosis. However, the HBV DNA, AST, ALT and Alb levels were significantly lower in patients with steatosis (all $P<0.05$ ).

Histological features of $\mathrm{HBeAg}$-negative $\mathrm{CHB}$ patients with or without steatosis were summarized in Table 2.

Table I Clinical characteristics and anthropometric indices of $\mathrm{HBeAg}$-negative $\mathrm{CHB}$ patients with and without steatosis

\begin{tabular}{llll}
\hline & CHB with steatosis & CHB without steatosis & $P$ value \\
\hline Number & 106 & 98 & 0.0078 \\
BMI $\left(\mathrm{kg} / \mathrm{m}^{2}\right)$ & $28.66 \pm 1.62$ & $20.74 \pm 1.01$ & 0.0371 \\
\hline FBG $(\mathrm{mmol} / \mathrm{L})$ & $6.79 \pm 0.84$ & $4.37 \pm 0.26$ & 0.0013 \\
\hline FINS $(\mathrm{U} / \mathrm{L})$ & $16.31 \pm 1.27$ & $11.62 \pm 0.84$ & 0.0064 \\
\hline TG $(\mathrm{mmol} / \mathrm{l})$ & $3.99 \pm 0.22$ & $2.65 \pm 0.10$ & 0.0216 \\
\hline TC $(\mathrm{mmol} / \mathrm{l})$ & $5.87 \pm 0.62$ & $3.70 \pm 0.57$ & 0.0014 \\
\hline ALT $(\mathrm{U} / \mathrm{L})$ & $110.82 \pm 21.59$ & $366.90 \pm 86.87$ & 0.0291 \\
AST $(\mathrm{U} / \mathrm{L})$ & $92.61 \pm 15.38$ & $157.62 \pm 23.31$ & 0.0116 \\
GGT $(\mathrm{U} / \mathrm{L})$ & $79.99 \pm 11.70$ & $48.63 \pm 6.72$ & 0.0268 \\
\hline ALP $(\mathrm{U} / \mathrm{L})$ & $157.514 \pm 14.72$ & $83.46 \pm 14.72$ & 0.0307 \\
\hline Alb $(\mathrm{g} / \mathrm{L})$ & $41.03 \pm 4.06$ & $47.89 \pm 4.73$ & 0.0053 \\
\hline Glb $(\mathrm{g} / \mathrm{L})$ & $41.84 \pm 11.73$ & $31.22 \pm 7.49$ & 0.0396 \\
\hline HOMA-IR & $5.86 \pm 1.03$ & $3.02 \pm 0.91$ & 0.0081 \\
\hline HBV DNA $\left(\log _{10}\right.$ copies/ml) & $3.29 \pm 1.08$ & $5.61 \pm 0.91$ & \\
\hline
\end{tabular}

Table 2 Histological features of $\mathrm{HBeAg}$-negative $\mathrm{CHB}$ patients with and without steatosis

\begin{tabular}{|c|c|c|c|}
\hline & CHB with steatosis (\%) & CHB without steatosis (\%) & $P$ value \\
\hline Number & 106 & 98 & \\
\hline \multicolumn{4}{|l|}{ Steatosis } \\
\hline F0 & 0 & $98(100)$ & \\
\hline $\mathrm{F} 1$ & $23(21.70)$ & 0 & \\
\hline F2 & $25(23.58)$ & 0 & \\
\hline F3 & $34(32.08)$ & 0 & \\
\hline F4 & $24(22.64)$ & 0 & \\
\hline \multicolumn{4}{|c|}{ Inflammation activity } \\
\hline G0 & 17(16.04) & $4(4.08)$ & 0.019 \\
\hline G1 & $51(48.11)$ & $13(13.27)$ & 0.000 \\
\hline G2 & $23(21.70)$ & $47(47.96)$ & 0.002 \\
\hline G3 & $15(14.15)$ & $34(34.69)$ & 0.010 \\
\hline \multicolumn{4}{|c|}{ Fibrosis stage } \\
\hline SO & $34(32.08)$ & $2(2.04)$ & 0.000 \\
\hline S1 & $49(46.22)$ & $15(15.31)$ & 0.000 \\
\hline S2 & $11(10.38)$ & $23(23.47)$ & 0.046 \\
\hline S3 & $6(5.66)$ & $42(42.85)$ & 0.000 \\
\hline S4 & $6(5.66)$ & $16(16.33)$ & 0.054 \\
\hline
\end{tabular}


The results of binary logistic regression are shown in Table 3. Among all indices and laboratory characteristics, FINS was the only characteristic that strongly associated with hepatic steatosis in HBeAg-negative CHB patients. The OR of FINS (every 1-unit increase) was 31.757 [95\% confidence interval (CI) 6.899 45.454, $P<0.001]$. The regression function for predicting hepatic steatosis among HBeAg-negative $\mathrm{CHB}$ patients could be defined as $\mathrm{P}=\mathrm{e}^{-240.827+17.165 \mathrm{FINS}} /\left(1+\mathrm{e}^{-240.827+17.165 \mathrm{FINS}}\right)$. The results of multivariate regression for hepatic inflammation are shown in Table 4. TG, GGT, Glb, and FINS were all associated with hepatic inflammation in each stage among HBeAg-negative CHB patients, but only FINS and $\mathrm{Glb}$ were strong predictors tested by likelihood ratio test $(P=0.014$, and $P=0.013$, respectively). Taken G0 stage as reference, each regression model could be expressed as follows: $\mathrm{G}_{1}$ :

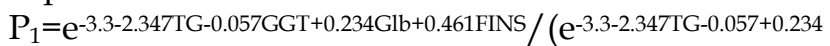

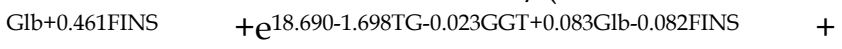

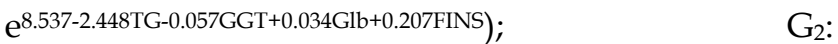
$\mathrm{P}_{2}=\mathrm{e}^{18.690-1.698 \mathrm{TG}-0.023 \mathrm{GGT}+0.083 \mathrm{Glb}-0.082 \mathrm{FINS}} /\left(\mathrm{e}^{-3.3-2.347 \mathrm{TG}-0.057+0.23}\right.$ 4 Glb+0.461FINS $\quad+\mathrm{e}^{18.690-1.698 \mathrm{TG}-0.023 \mathrm{GGT}+0.083 \mathrm{Glb}-0.082 \mathrm{FINS}+}+$

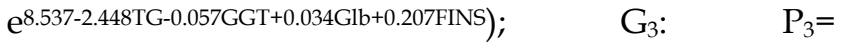

$\mathrm{e}^{8.537-2.448 \mathrm{TG}-0.057 \mathrm{GGT}+0.034 \mathrm{Glb}+0.207 \mathrm{FINS}} /\left(\mathrm{e}^{-3.3-2.347 \mathrm{TG}-0.057+0.234 \mathrm{Glb}+}\right.$

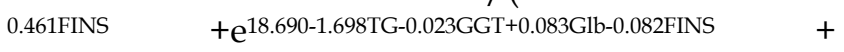

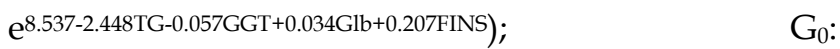
$\mathrm{P}_{0}=1 /\left(\mathrm{e}^{-3.3-2.347 \mathrm{TG}-0.057+0.234 \mathrm{Glb}+0.461 \text { FINS }}\right.$

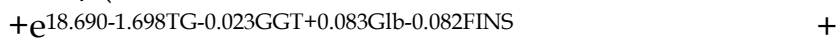
$\mathrm{e}^{8.537-2.448 \mathrm{TG}-0.057 \mathrm{GGT}+0.034 \mathrm{Glb}+0.207 \mathrm{FINS})}$. Similarly, the results of multivariate regression for hepatic fibrosis were shown in Table 5. BMI and TC were strongly predictors of hepatic fibrosis among HBeAg-negative CHB patients tested by likelihood ratio test $(P=0.033$ and $P=0.025$, respectively). Regression function for each stage could be expressed as follows: $S_{1}$ : $\mathrm{P}_{1}=\mathrm{e}^{-22.942+0.087 \mathrm{BMI}+4.203 \mathrm{TC}} /\left(\quad \mathrm{e}^{-22.942+0.087 \mathrm{BMI}+4.203 \mathrm{TC}+}\right.$

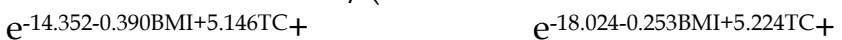
$\mathrm{e}^{-39.445+0.199 \mathrm{BMI}+6.543 \mathrm{TC})} ; \quad \mathrm{S}_{2}: \quad \mathrm{P}_{2}=\mathrm{e}^{-14.352-0.390 \mathrm{BMI}+5.146 \mathrm{TC}} /($

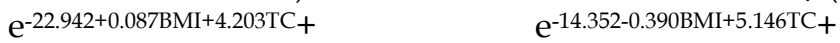
$\left.\mathrm{e}^{-18.024-0.253 \mathrm{BMI}+5.224 \mathrm{TC}+} \quad \mathrm{e}^{-39.445+0.199 \mathrm{BMI}+6.543 \mathrm{TC}}\right) ; \quad \mathrm{S}_{3}: \quad \mathrm{P}_{3}=$

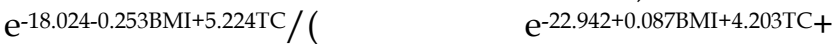
$\mathrm{e}^{-14.352-0.390 \mathrm{BMI}+5.146 \mathrm{TC}+} \quad \mathrm{e}^{-18.024-0.253 \mathrm{BMI}+5.224 \mathrm{TC}+}+$ $\mathrm{e}^{-39.445+0.199 \mathrm{BMI}+6.543 \mathrm{TC})} ; \quad \mathrm{S}_{4}: \quad \mathrm{P}_{4}=\mathrm{e}^{-39.445+0.199 \mathrm{BMI}+6.543 \mathrm{TC}} /($

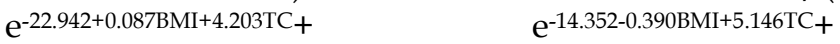

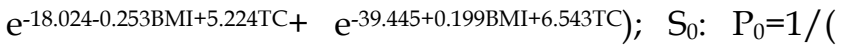
$\mathrm{e}^{-22.942+0.087 \mathrm{BMI}+4.203 \mathrm{TC}+} \quad \mathrm{e}^{-14.352-0.390 \mathrm{BMI}+5.146 \mathrm{TC}+}$

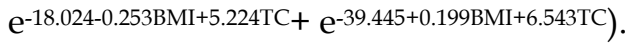

Table 3 Binary logistic regression analysis was performed to screen predictors for hepatic steatosis in HBeAg-negative CHB patients

\begin{tabular}{|l|l|l|l|l|l|}
\hline & $B$ & $S E$ & Wald $\chi^{2}$ & $P$ & OR \\
\hline FINS & 17.165 & 1432.081 & 19.148 & $1.210 \mathrm{E}-3$ & 31.757 \\
\hline Constant & -240.827 & 20161.091 & 20.373 & $6.374 \mathrm{E}-6$ & 0.107 \\
\hline Cox and Snell $R^{2}$ & 0.750 & & & \\
\hline Nagelkerke $R^{2}$ & 0.830 & & & \\
\hline Overall percentage & $82 \%$ & & & \\
\hline
\end{tabular}

Table 4 Multinomial logistic regression analysis was performed to screen predictors for hepatic inflammation grades in $\mathrm{HBeAg}$-negative $\mathrm{CHB}$ patients. Categorical variables were defined as follows: $\mathrm{G}_{0}: 0, \mathrm{G}_{1}: \mathrm{I}, \mathrm{G}_{2}: 2, \mathrm{G}_{3}: 3$.

\begin{tabular}{|c|c|c|c|c|c|c|c|}
\hline & G & Wald $x^{2}$ & $P$ & OR & $95 \% C I$ & Cox and Snell $R^{2}$ & Model fitting test \\
\hline \multirow[t]{3}{*}{ TG } & 1 & 7.004 & 0.008 & 0.096 & $0.017 \sim 0.544$ & 0.271 & $P=0.001$ \\
\hline & 2 & 2.547 & 0.111 & 0.183 & $0.023 \sim 1.473$ & 0.271 & $P=0.001$ \\
\hline & 3 & 4.331 & 0.037 & 0.086 & $0.009 \sim 0.867$ & 0.271 & $P=0.001$ \\
\hline \multirow[t]{3}{*}{ GGT } & 1 & 4.330 & 0.037 & 0.945 & $0.896 \sim 0.997$ & - & - \\
\hline & 2 & 0.620 & 0.431 & 0.977 & $0.922 \sim 1.035$ & - & - \\
\hline & 3 & 3.246 & 0.072 & 0.944 & $0.887 \sim 1.005$ & - & - \\
\hline \multirow[t]{3}{*}{ Glb } & 1 & 5.542 & 0.019 & 1.263 & $1.040 \sim 1.535$ & - & - \\
\hline & 2 & 0.570 & 0450 & 1.087 & $0.875 \sim 1.350$ & - & - \\
\hline & 3 & 0.076 & 0.782 & 1.034 & $0.814 \sim 1.314$ & - & - \\
\hline \multirow[t]{3}{*}{ FINS } & 1 & 0.955 & 0.328 & 1.585 & $0.629 \sim 3.995$ & - & - \\
\hline & 2 & 2.681 & 0.102 & 0.435 & $0.161 \sim 1.178$ & - & - \\
\hline & 3 & 0.137 & 0.711 & 1.230 & $0.411 \sim 3.677$ & - & - \\
\hline
\end{tabular}


Table 5 Multinomial logistic regression analysis was performed to screen predictors for hepatic fibrosis stages in HBeAg-negative CHB patients. Categorical variables were defined as follows: S0: 0, SI: I, S2: 2, S3: 3, S4: 4.

\begin{tabular}{lllllllll}
\hline S & Index & Wald $\chi^{2}$ & $P$ & OR & $95 \% C I$ & Cox and Snell $R^{2}$ & Model $P$ \\
S1 & BMI & 0.019 & 0.890 & 1.090 & $0.320 \sim 3.717$ & 0.170 & \\
& TC & 1.796 & 0.180 & 66.911 & $0.143 \sim 31279.998$ & & 0.011 \\
S2 & BMI & 0.404 & 0.525 & 0.677 & $0.203 \sim 2.256$ & 0.170 & \\
& TC & 2.720 & 0.099 & 171.712 & $0.379 \sim 77702.459$ & & 0.170 & \\
S3 & BMI & 0.174 & 0.677 & 0.776 & $0.236 \sim 2.556$ & 0.11 & \\
& SC & 2.838 & 0.092 & 185.683 & $0.426 \sim 80963.996$ & & 0.170 & \\
& TC & BMI & 0.098 & 0.754 & 1.220 & $0.352 \sim 4.220$ & 0.011 \\
& TC & 4.314 & 0.038 & 694.314 & $1.446 \sim 333294.121$ & & \\
\hline
\end{tabular}

\section{Discussion}

HBeAg-negative and HBeAg-positive hepatitis are two different types of chronic hepatitis B with distinct clinical features (9). The prevalence of HBeAg-negative hepatitis as well as non-alcoholic fatty liver disease (NAFLD) has been increasing in the past decades $(4,10)$. Increasing studies on chronic hepatitis $C$ with hepatic steatosis have been conducted, but little is known about $\mathrm{CHB}$ with steatosis. Hepatic steatosis may have different influences on the liver affected by other diseases. Therefore, it cannot always be considered as a "benign" condition and simply ignored. On the contrary, it has to be recognized as a "co-factor" capable of affecting the gravity and progression and also therapeutic perspectives of liver diseases.

We compared the clinical and histological characteristics between $\mathrm{HBeAg}$-negative $\mathrm{CHB}$ patients with and without steatosis, and the results demonstrated significant increases in BMI, FBG, FINS, TG, TC, GGT, ALP, Glb and HOMA-IR in patients with steatosis, implying that obesity, diabetes and hyperlipemia appeared to be the risk factors in patients with steatosis, and insulin resistance might play an important role (12). HBeAg-negative $\mathrm{CHB}$ is characterized by low spontaneous remission, frequent ALT flare, easy progression to cirrhosis, low HBV DNA titer and curative difficulty, and thus hepatic steatosis will definitely increase the difficulty of therapy in HBeAg-negative HB patients (13).

In comparison to $\mathrm{HBeAg}$-negative $\mathrm{CHB}$ with hepatic steatosis, the ALT, AST and HBV-DNA levels were higher in patients without steatosis, indicating that the ALT and AST flares may be associated with HBV DNA titer in our study, while in patients with hepatic steatosis, these parameters are more likely related to hepatic steatosis. Thus, for the treatment of HBeAg-negative $\mathrm{CHB}$ with hepatic steatosis, in addition to antivirus therapy and liver protection therapy, insulin resistance reduction, lipid modulation, diet restriction and exercise for prevention and control of risk factors are also important. Some HBeAg-negative
CHB patients with hepatic steatosis may even progress into fibrosis and cirrhosis (14). In HBeAg-negative CHB patients with hepatic steatosis, the activity of hepatic inflammation may be associated with NAFLD in the presence of slightly high ALT level and low HBV DNA level. Clinically, it is very difficult to conclude whether hepatitis is from steatosis and/or HBV infection through detecting ALT, HBeAg and HBV DNA levels (15). Under such condition, in addition to detection of the ALT, HBeAg and HBV DNA levels, BMI, FBG, FINS, TG, TC, GGT, ALP, Glb and HOMA-IR are also critical for diagnosis. If these parameters are abnormal, liver biopsy is strongly recommended in order to assess histology and prognosis. Our study demonstrated the significance of liver biopsy in determining the causes of high ALT levels. The most important limitation of this study is the lack of long-term follow-up and evaluation of response to antiviral therapy in HBeAg-negative $\mathrm{CHB}$ patients with steatosis.

\section{Acknowledgment}

This work was supported by the grant from Science and Technology Commission of Shanghai Municipality (No.054119618) and Technology Fund of Zhangzhou (No. Z04094). We appreciate Dr. Qianglin Duan from Tongji Hospital of Tongji University for critical reading of the manuscript.

\section{Conflict of Interest}

The authors have declared that no conflict of interest exists.

\section{References}

1. Hadziyannis SJ, Papatheodoridis GV. Hepatitis B e antigen-negative chronic hepatitis $\mathrm{B}$ : natural history and treatment. Semin Liver Dis. 2006; 26:130-41.

2. Bondini S, Kallman J, Wheeler A, et al. Impact of non-alcoholic fatty liver disease on chronic hepatitis B. Liver Int. 2007;27:607-11.

3. Browning JD, Szczepaniak LS, Dobbins R, et al. Prevalence of hepatic steatosis in an urban population in the United States: impact of ethnicity. Hepatology. 2004;40:1387-95. 
4. Angulo P. Nonalcoholic fatty liver disease. Rev Gastroenterol Mex. 2005; 70:52-6.

5. Bondini S, Younossi ZM. Non-alcoholic fatty liver disease and hepatitis C infection. Minerva Gastroenterol Dietol. 2006; 52:135-43.

6. Lok AS, McMahon BJ. Chronic hepatitis B. Hepatology. 2007; 45:507-39.

7. Matthews DR, Hosker JP, Rudenski AS, et al. Homeostasis model assessment: insulin resistance and bcell function from fasting plasma glucose and insulin concentrations in man. Diabetologia. 1985; 28:412-9.

8. Farrell GC, Chitturi S, Lau GK, et al. Guidelines for the assessment and management of non-alcoholic fatty liver disease in the Asia-Pacific region: executive summary. $J$ Gastroenterol Hepatol. 2007; 22: 775-7.

9. Pungpapong S, Kim WR, Poterucha JJ. Natural history of hepatitis B virus infection: an update for clinicians. Mayo Clin Proc. 2007; 82:967-75.

10. Keeffe EB, Marcellin P. New and emerging treatment of chronic hepatitis B. Clin Gastroenterol Hepatol. 2007; 5:285-94.

11. Persico M, Iolascon A. Steatosis as a co-factor in chronic liver diseases. World J Gastroenterol. 2010;16:1171-6.

12. Bondini S, Kallman J, Wheeler A, et al. Impact of non-alcoholic fatty liver disease on chronic hepatitis B. Liver Int. 2007; 27:607-611.

13. Gordon A, McLean CA, Pedersen JS, et al. Hepatic steatosis in chronic hepatitis B and C: Predictors, distribution and effect on fibrosis. J Hepatol. 2005; 43:38-44.

14. Ekstedt M, Franzén LE, Mathiesen UL, et al. Long-term follow-up of patients with NAFLD and elevated liver enzymes. Hepatology. 2006; 44:865-73.

15. Demir K, Akyuz F, Ozdil S. What is the reason of elevated alanine aminotransferase level in $\mathrm{HBeAg}$ negative patients with low viremia: NAFLD or chronic hepatitis? Ann Hepatol. 2007; 6:92-6. 